\title{
PROSPECTS FOR DEEPENING TRADE AND ECONOMIC RELATIONS BETWEEN GEORGIA AND THE EUROPEAN UNION
}

\section{EKATERINE UDESIANI}

PhD student

Ivane Javakhishvili Tbilisi State University, Georgia

eudesiani@gmail.com

Abstract. The association agreement aims at developing political and economic relations between European Union and Georgia. By association agreement Georgia has received a huge opportunity to become a competitive member of the European Union market and with Deep and Comprehensive Free Trade Area (DCFTA) preferences, introduce Georgian products at the world market with zero customs charge. DCFTA includes a wide range of issues concerning trade and considers gradual approximation of Georgian legislation to EU legislation. DCFTA implies liberalization of both goods and service trade. It gives Georgia a chance to receive all four EU freedoms from EU internal market: goods, service, capital and free movement of persons on EU territory (based on the liberalization of visa regime).

And yet, what is DCFTA for Georgia: A special trade regime which, if used correctly, can significantly improve investment potential of Georgia and steadily develop the economy of the country; The preference which can ensure the growth of competitiveness of Georgian products and the demand for products produced in Georgia; The agreement which gives the Georgian side a very good chance to increase the volume of export with EU countries and significantly improve negative balance of trade; The regime which gives Georgian products a chance to enter EU with zero Customs charge and establish themselves at one of the largest world markets, which unites 500 million consumers and 21 million small and medium enterprises.

KEYWORDS: ASSOCIATION AGREEMENT; TRADE AND ECONOMIC RELATIONS; DIAGONAL CUMULATION MODE; PANDEMIC; GLOBAL CRISIS.

For citation: Udesiani, E., (2021). Prospects for Deepening Trade and Economic Relations Between Georgia and the European Union. Globalization and Business, №11, 50-55. (In Georgian). https://doi.org/10.35945/gb.2021.11.006 


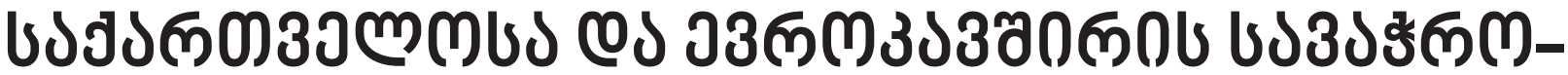

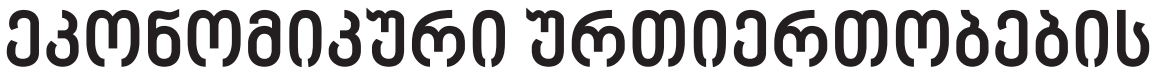

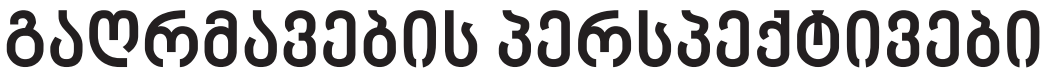

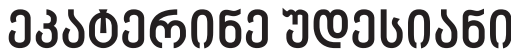

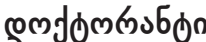

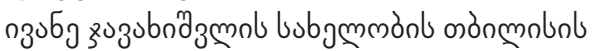

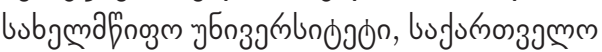

eudesiani@gmail.com

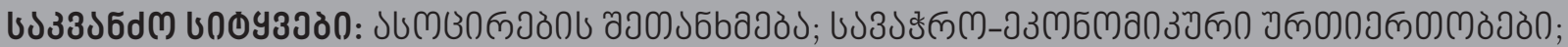

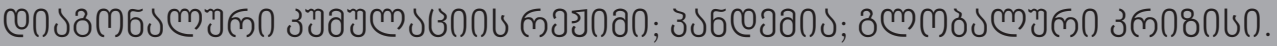

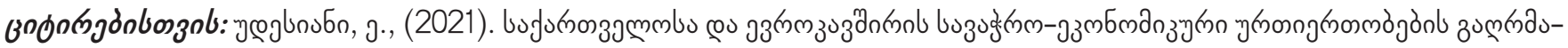

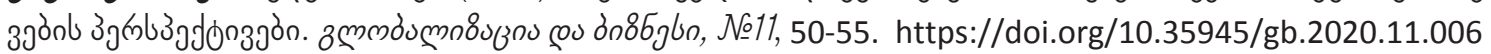

\section{ฆอง১З১ल0}

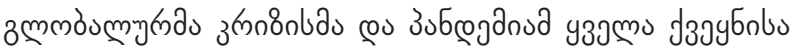

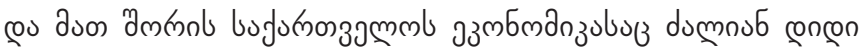

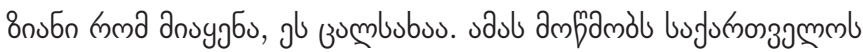
2020 fmmnl auz

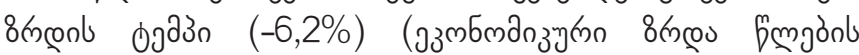

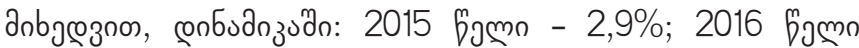

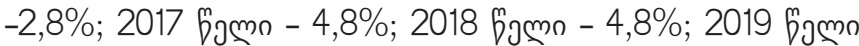

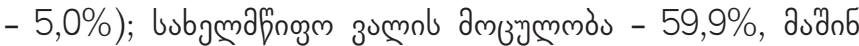

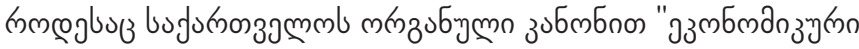

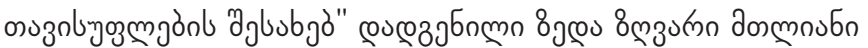

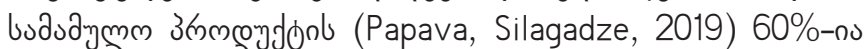

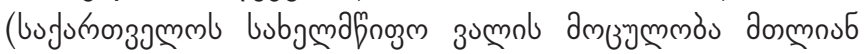

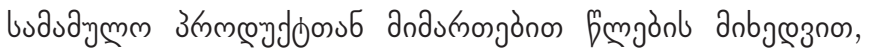

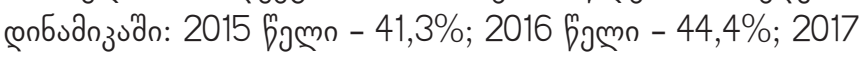

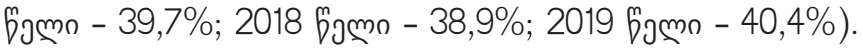

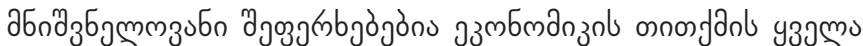

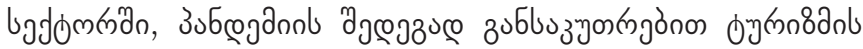

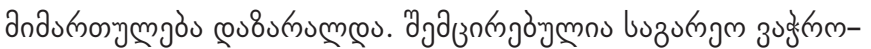

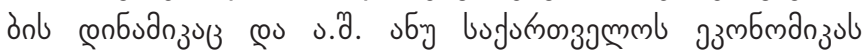

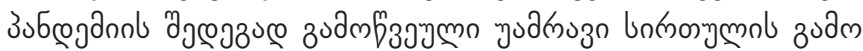

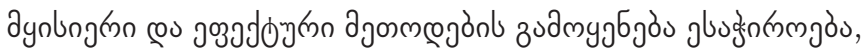

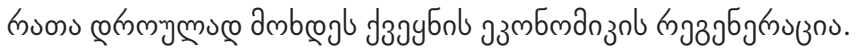

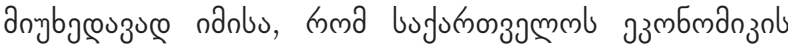

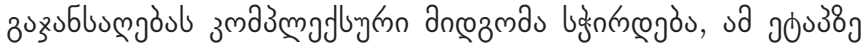

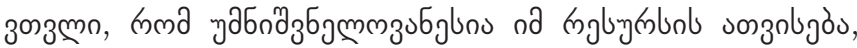

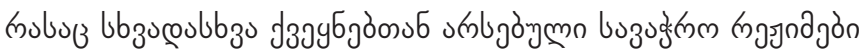

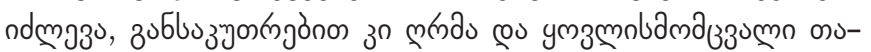

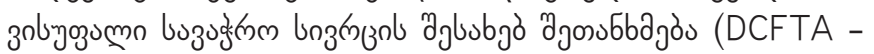

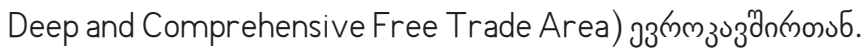

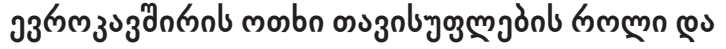

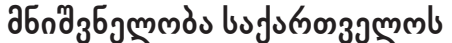

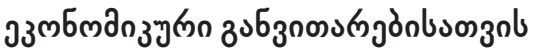

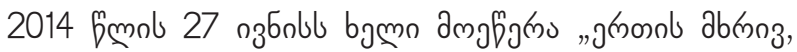

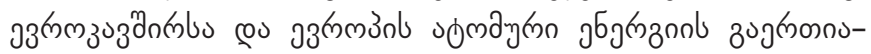

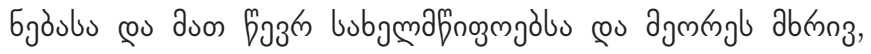

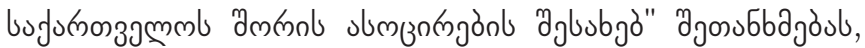

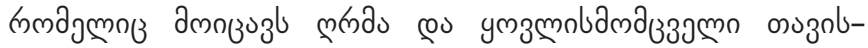

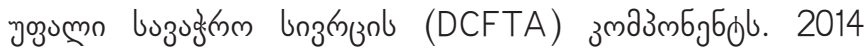

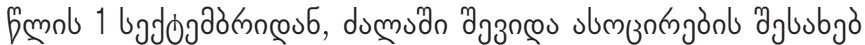

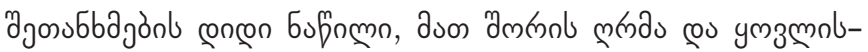

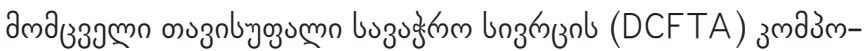

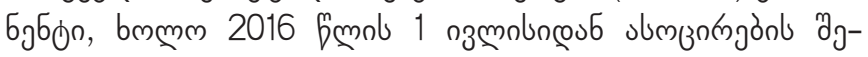

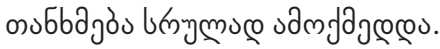

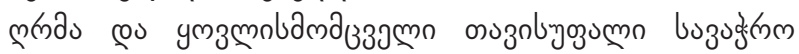

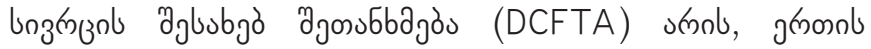

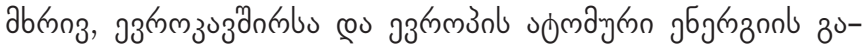

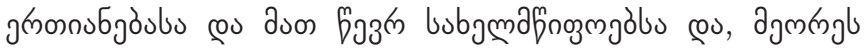

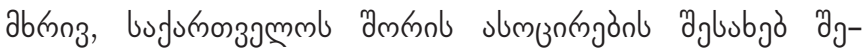

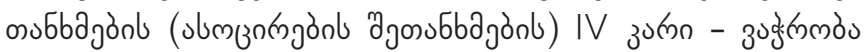

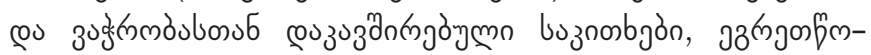

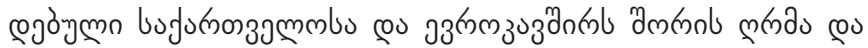

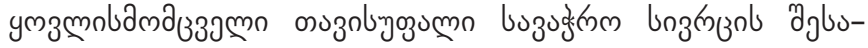

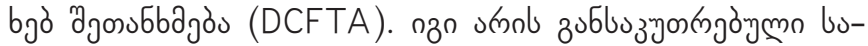

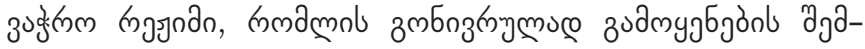

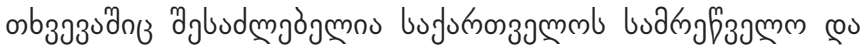

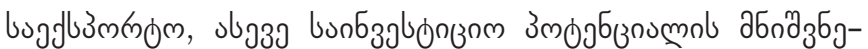

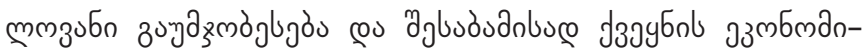
zol a

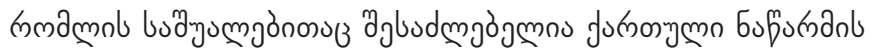




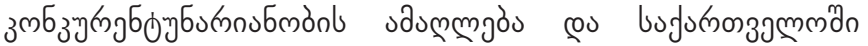

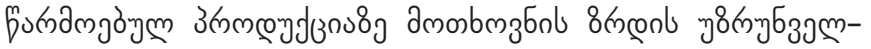

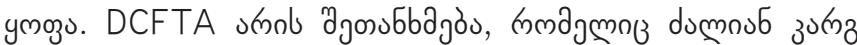

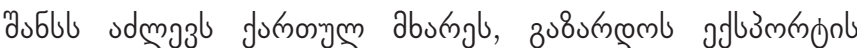

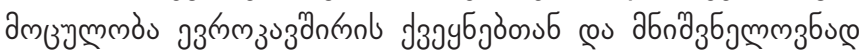

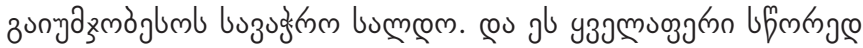

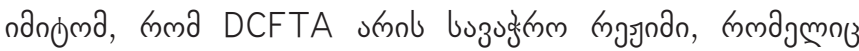

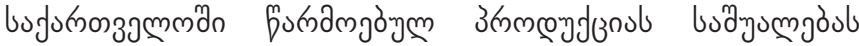

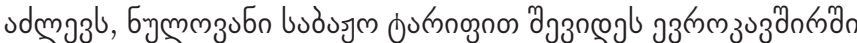

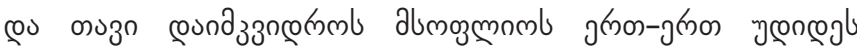

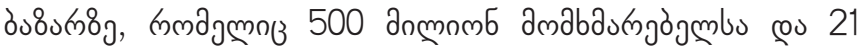

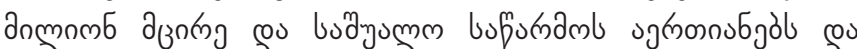

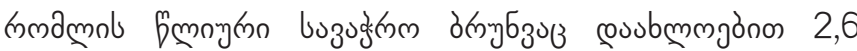

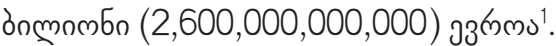

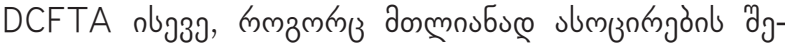

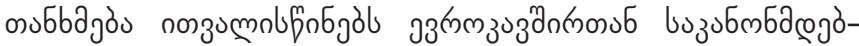

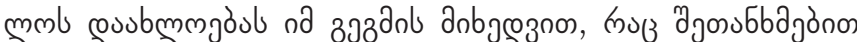

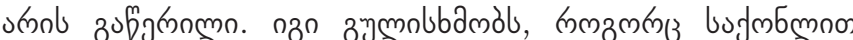

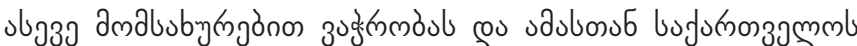

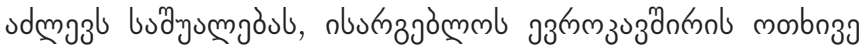

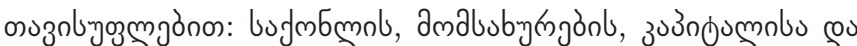

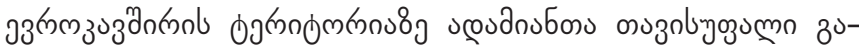

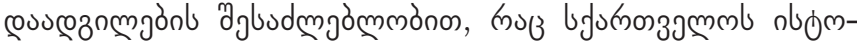
mnymo дmbsümzutros.

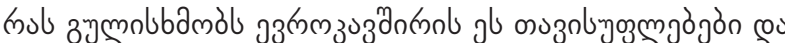

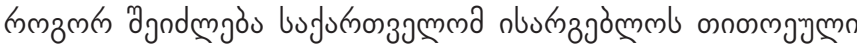
asonzubnom?

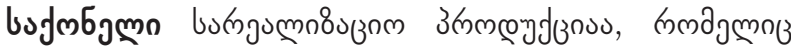

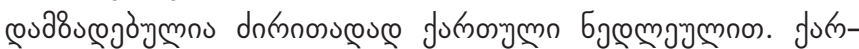

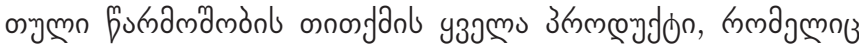

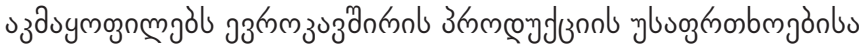

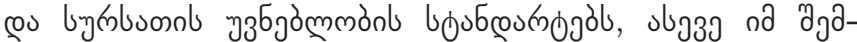

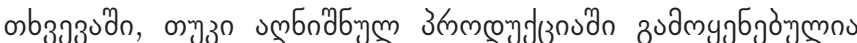

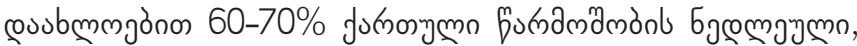

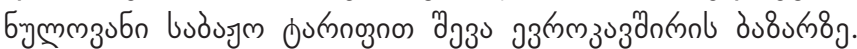

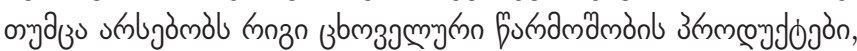

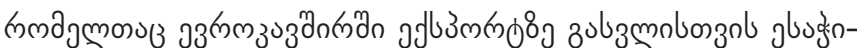

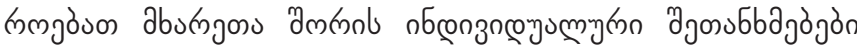

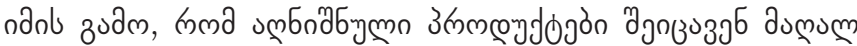

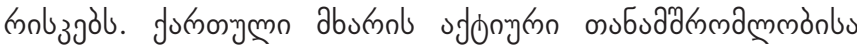

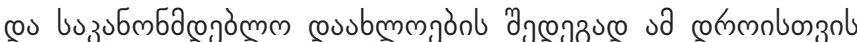

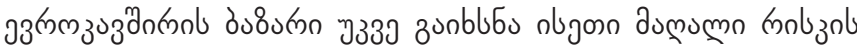

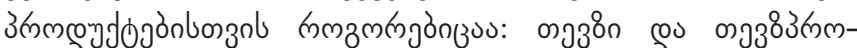

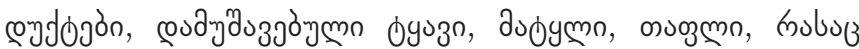

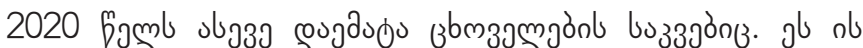

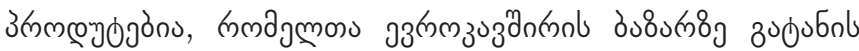

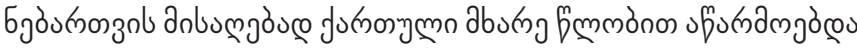

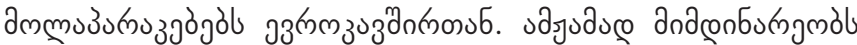

${ }^{1}$ эз info/index_en

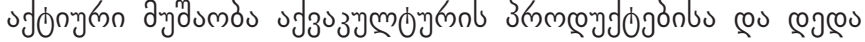
o3ydz

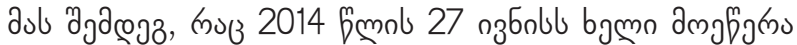

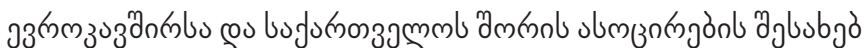

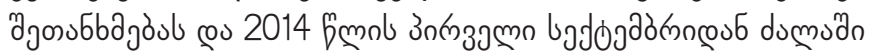

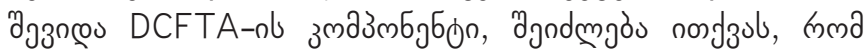

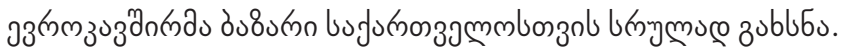

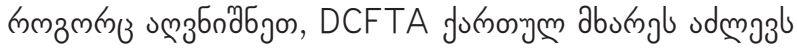

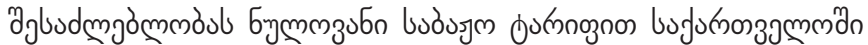

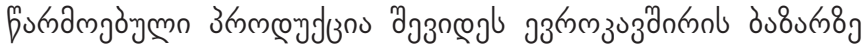

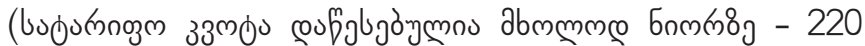

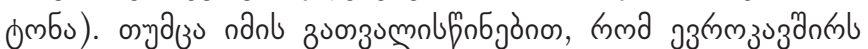

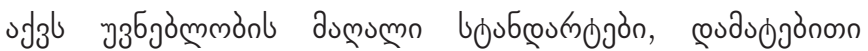

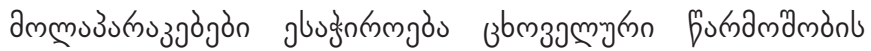

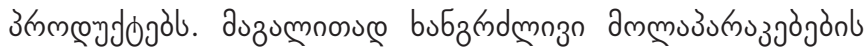

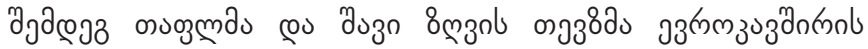
ju

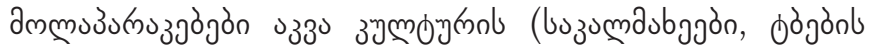

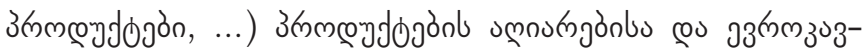

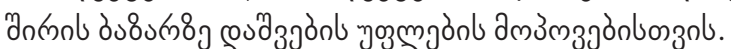

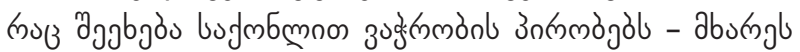

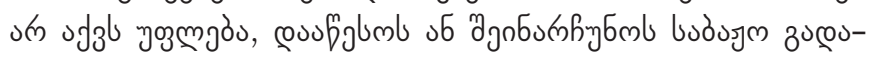

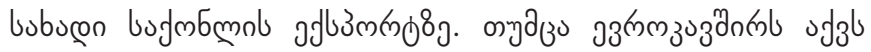

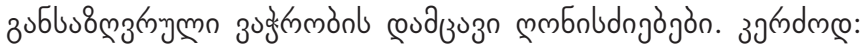

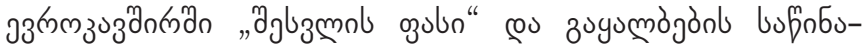

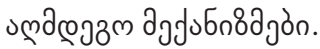

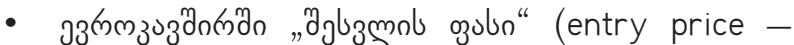
ad valorem free) dm dagejal 28 bupumnogm bu88j - on

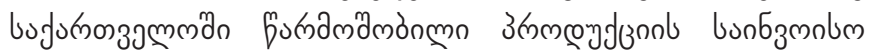

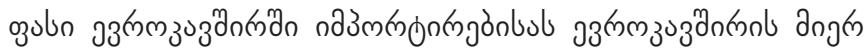

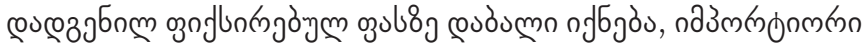

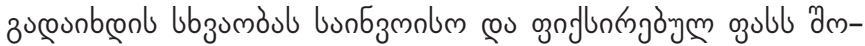

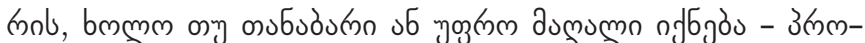

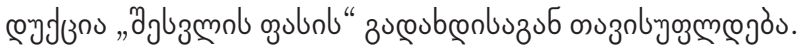

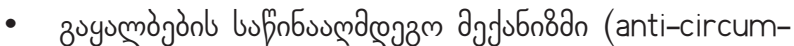

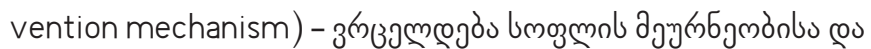

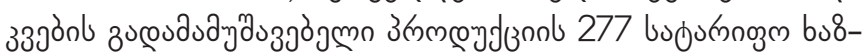

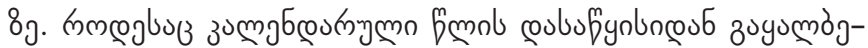

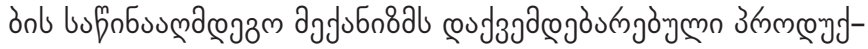

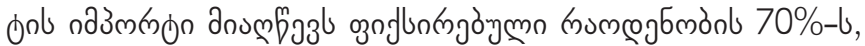

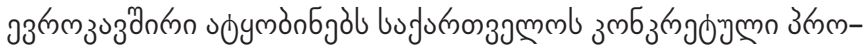

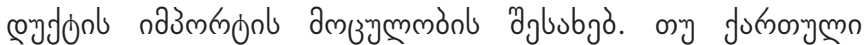

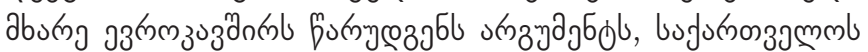

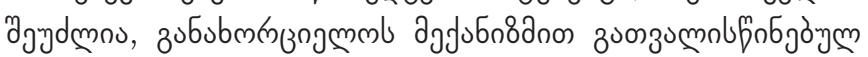

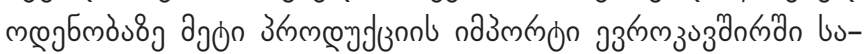
bujm zucoububuconb zukngag ${ }^{2}$.

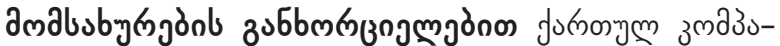

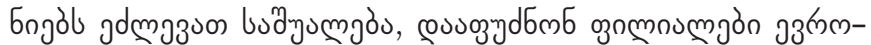

2 http://www.dcfta.gov.ge/ 


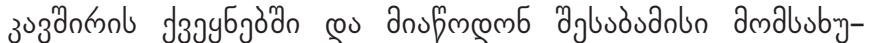

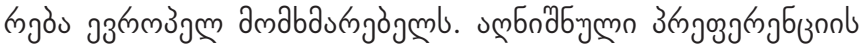

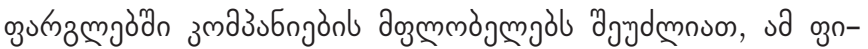

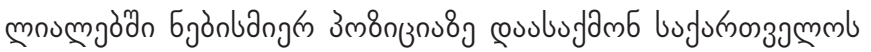
anjumudgaòn;

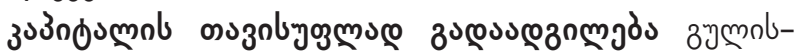

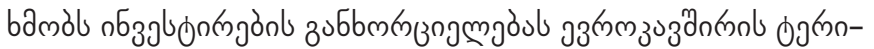

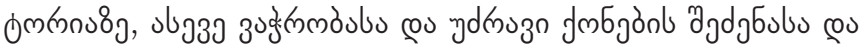

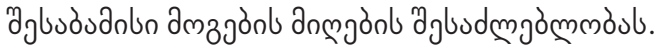

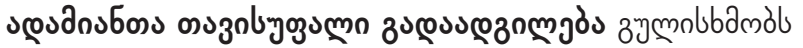

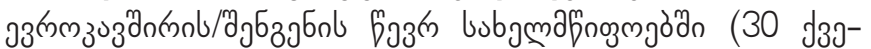

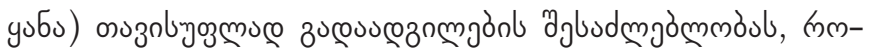

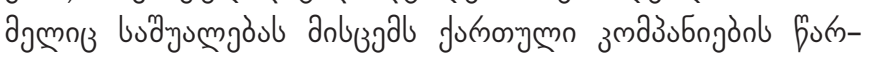

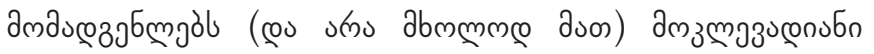

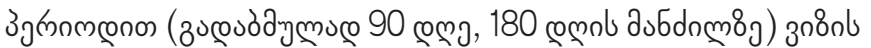

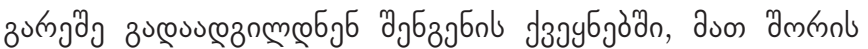

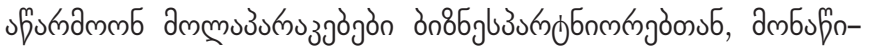

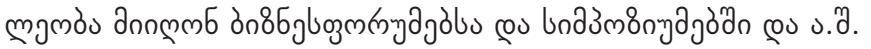

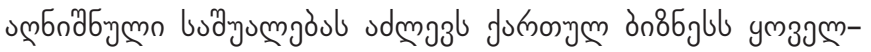

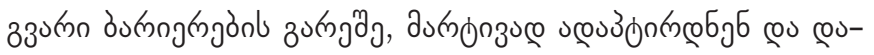

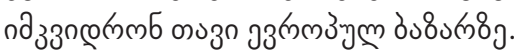

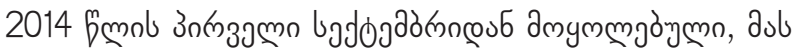

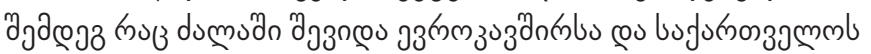

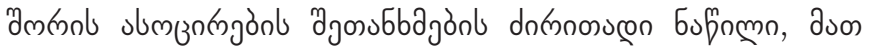

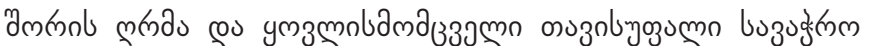

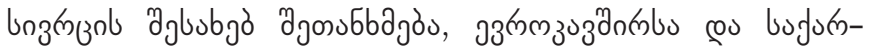

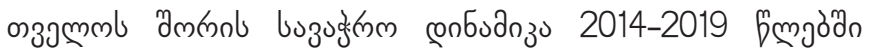

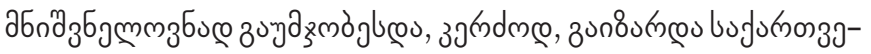

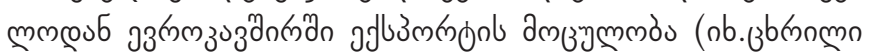

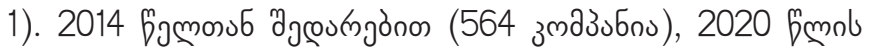

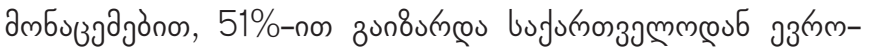

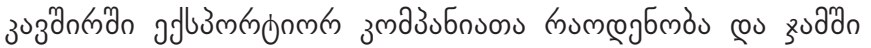

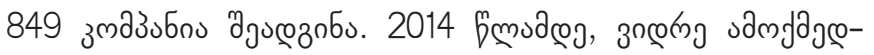

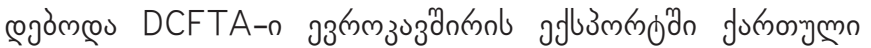

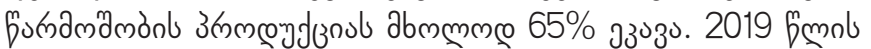

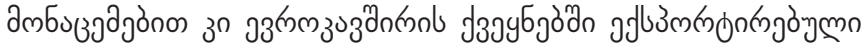

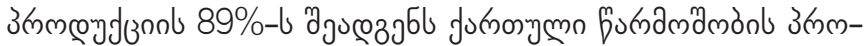

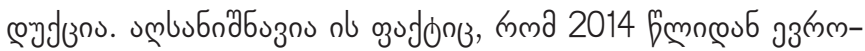

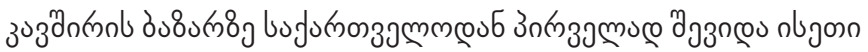

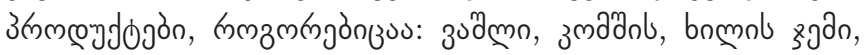

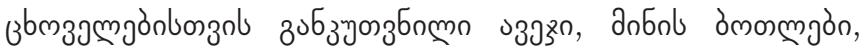

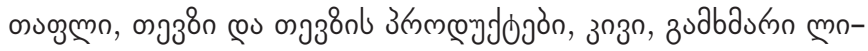
ambn, byk

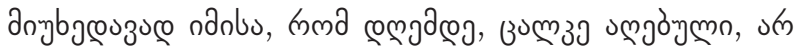

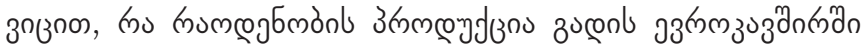

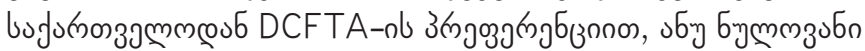

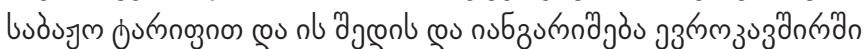
ammnuton ajlusminonb as

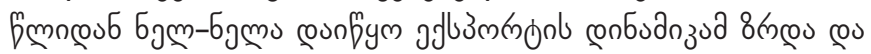

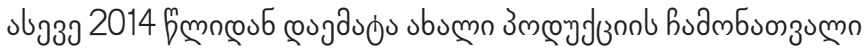

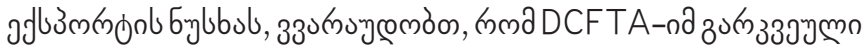

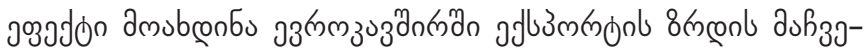

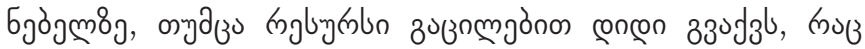

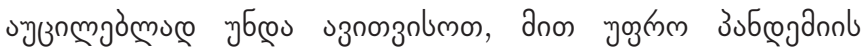

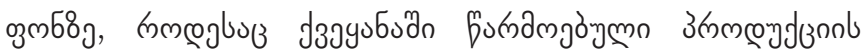

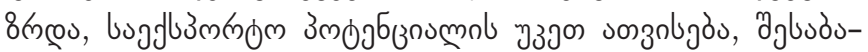

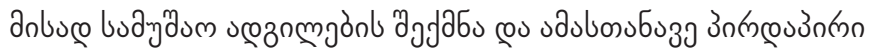

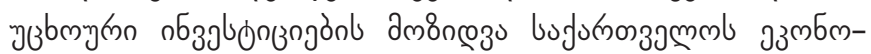

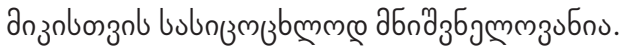

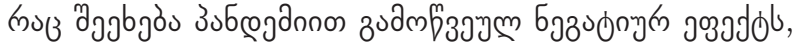

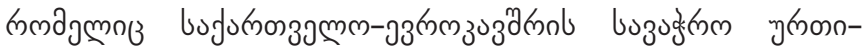

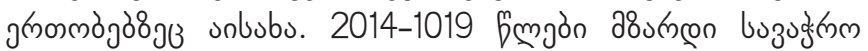

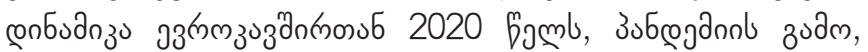

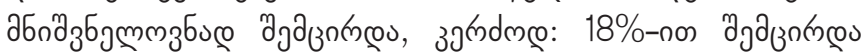

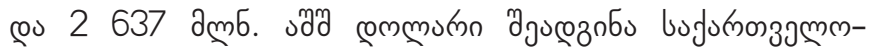

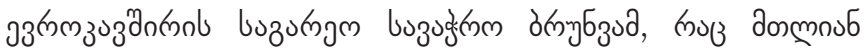

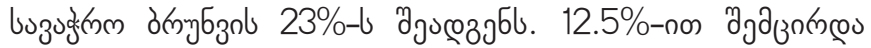

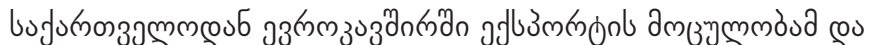

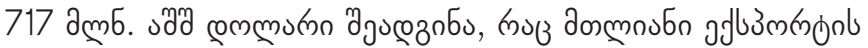

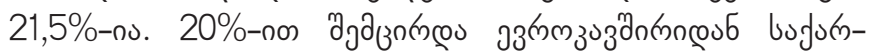

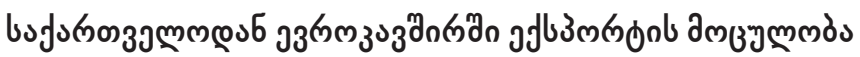

\begin{tabular}{|c|c|c|c|}
\hline \multicolumn{4}{|c|}{ 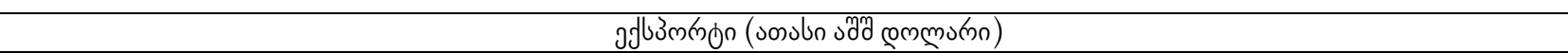 } \\
\hline & 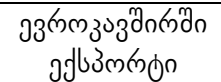 & $\begin{array}{l}\text { donmaubn } \\
\text { jلsusmonon }\end{array}$ & 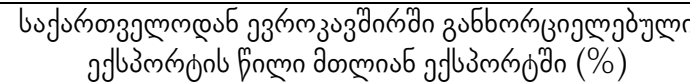 \\
\hline 2014 & $624,201.4$ & $2,861,045.2$ & 21.8 \\
\hline 2015 & $644,729.9$ & $2,204,188.6$ & 29.3 \\
\hline 2016 & $565,675.0$ & $2,117,149.2$ & 26.7 \\
\hline 2017 & $655,435.8$ & $2,745,743.8$ & 23.9 \\
\hline 2018 & $729,224.3$ & $3,379,732.4$ & 21.6 \\
\hline 2019 & $819,242.4$ & $3,798,449.5$ & 21.6 \\
\hline 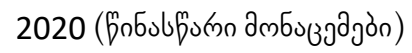 & $716,973,4$ & $3,342,142,2$ & 21.5 \\
\hline
\end{tabular}

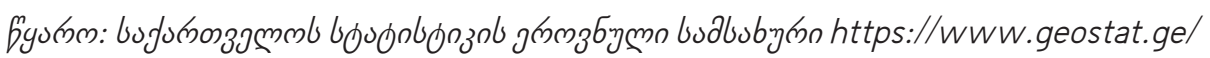




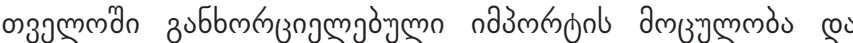

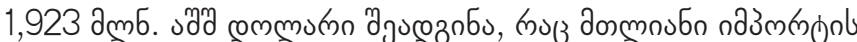
24\%-no

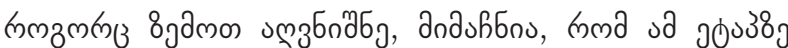

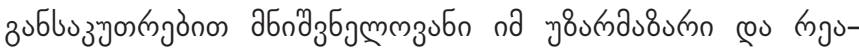

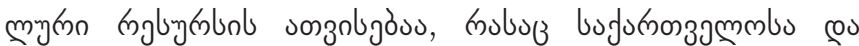

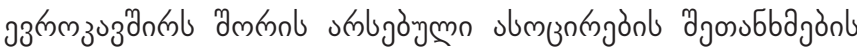

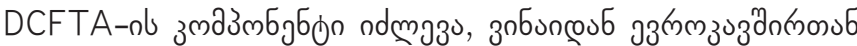

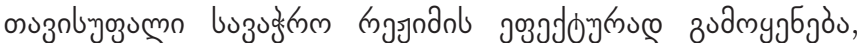
১а

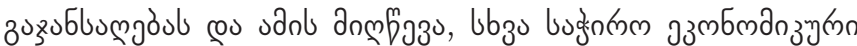

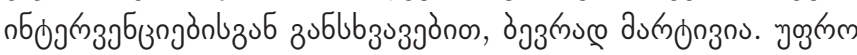

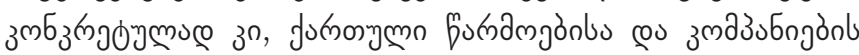

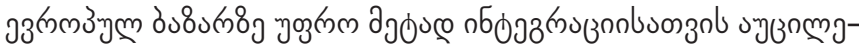

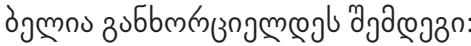

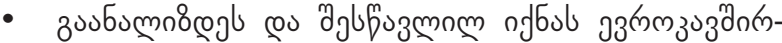

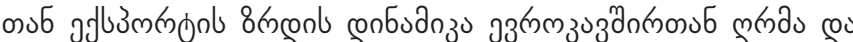

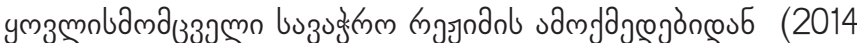

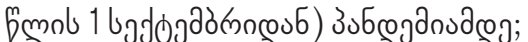

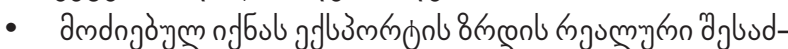

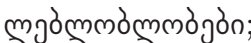

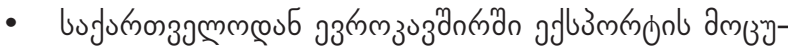

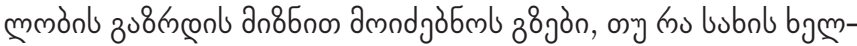

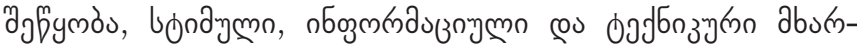

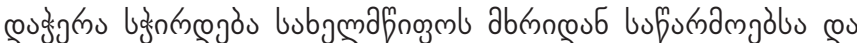
8 mzucue on85glb;

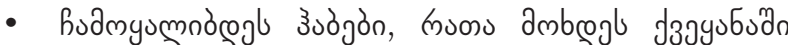

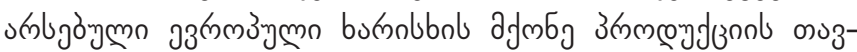

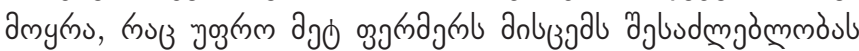

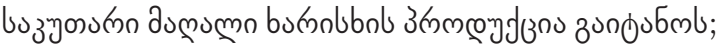

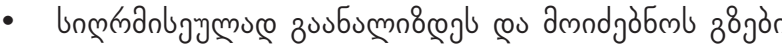

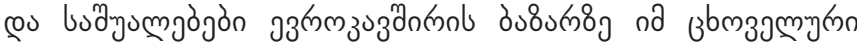

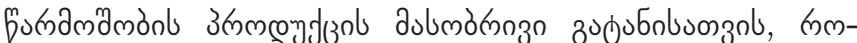

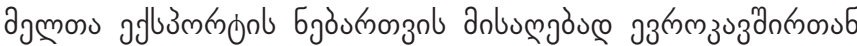

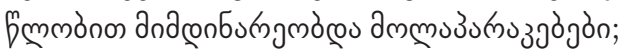

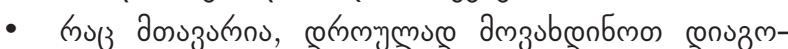

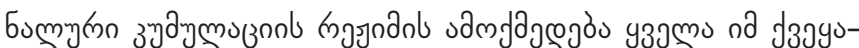

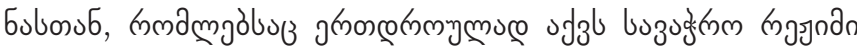

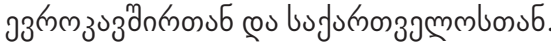

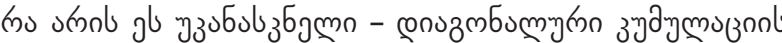

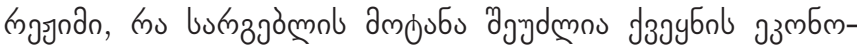

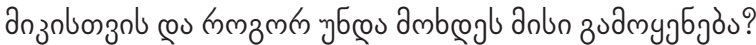

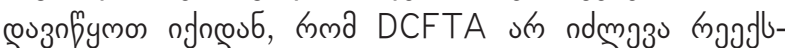

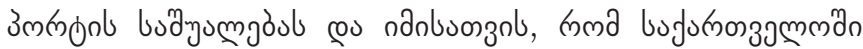

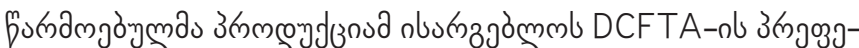

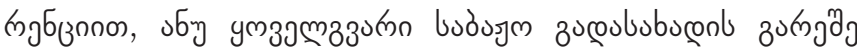

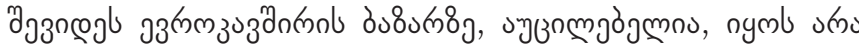

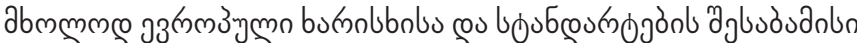

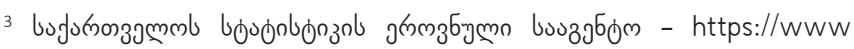
geostat.ge/ka
}

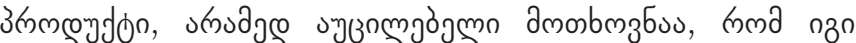

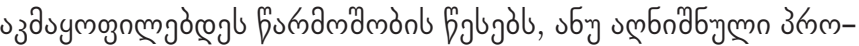

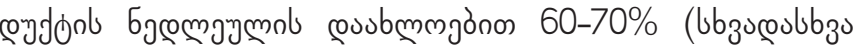

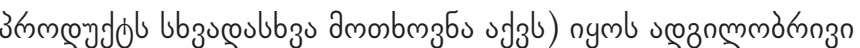

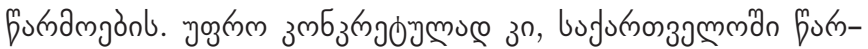

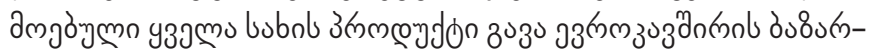

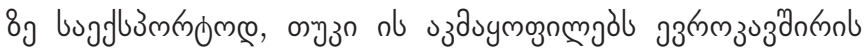

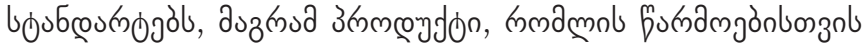

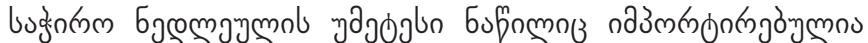

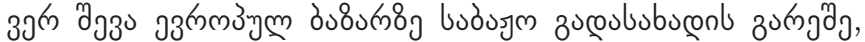
د6y 3gr

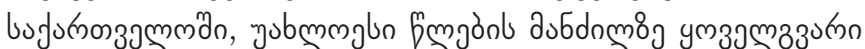

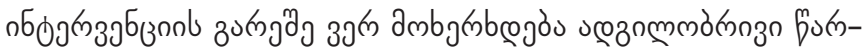

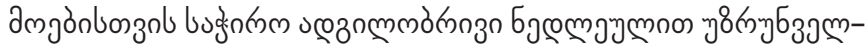

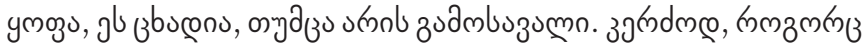
8gamon uмзб

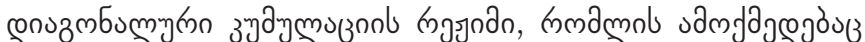

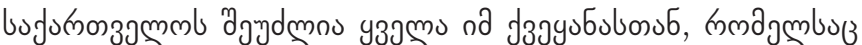

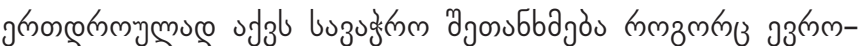

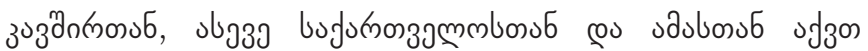

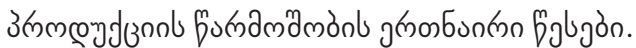

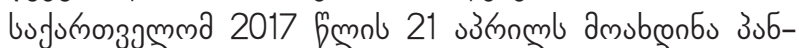

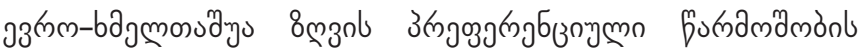

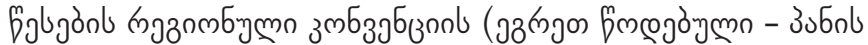

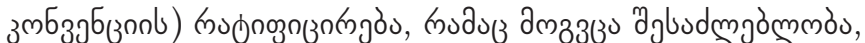

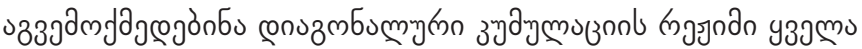

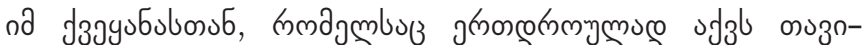

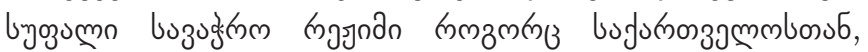

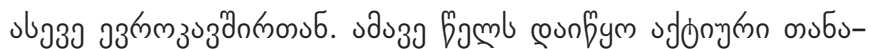

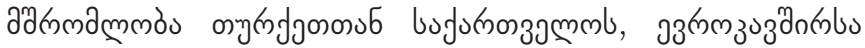

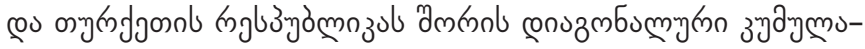

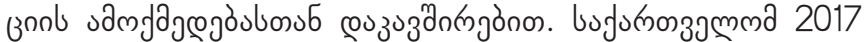

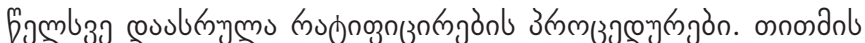

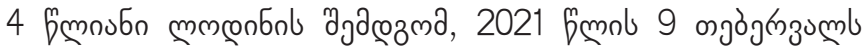

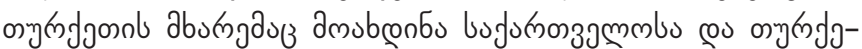

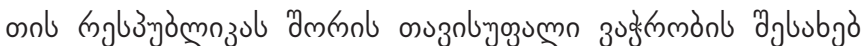

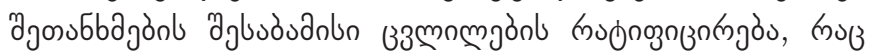

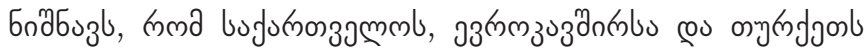

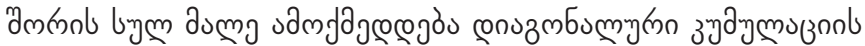

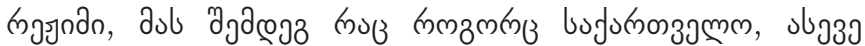

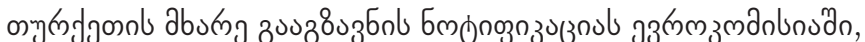

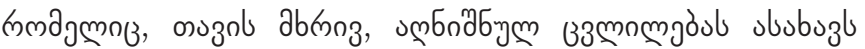

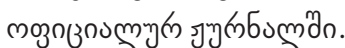

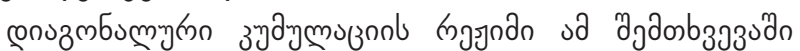

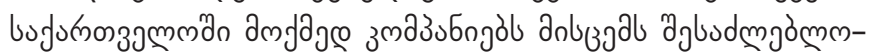

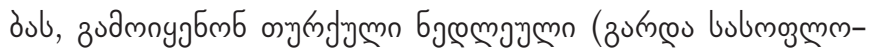

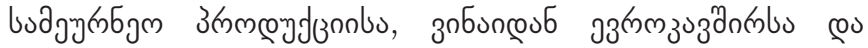

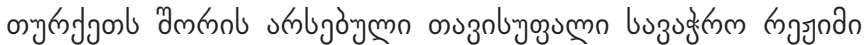

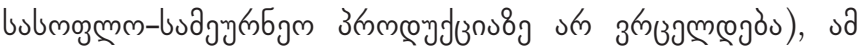

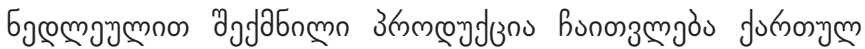




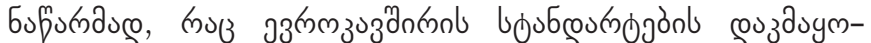

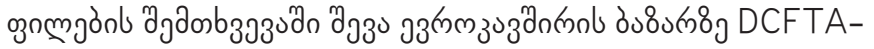

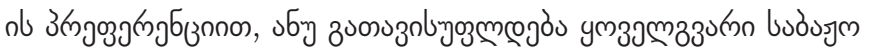

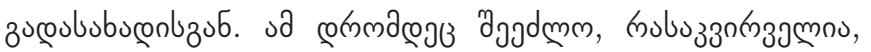

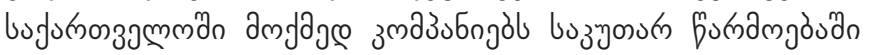

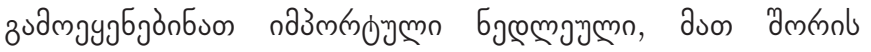

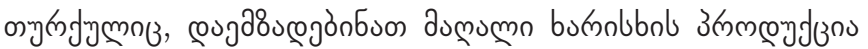

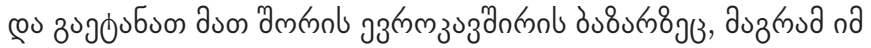

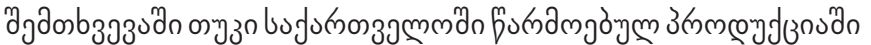

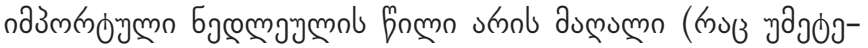

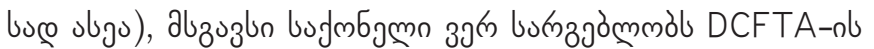

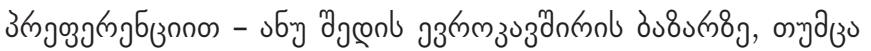

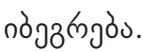

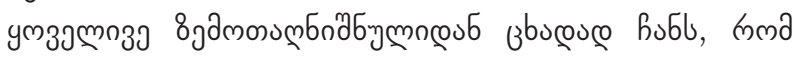

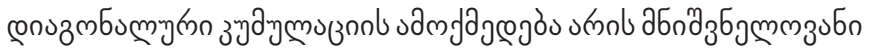

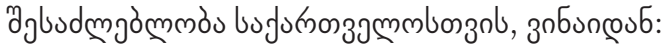

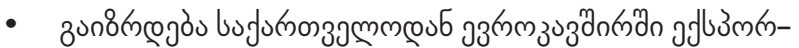

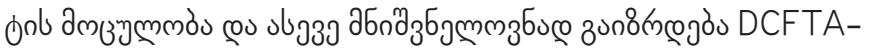

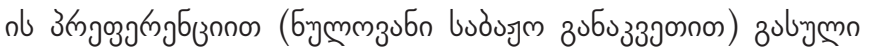

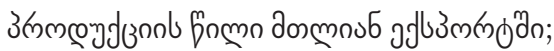

- bymb ànfy

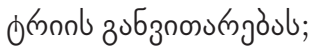

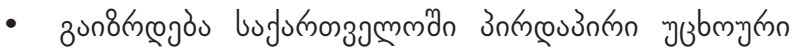

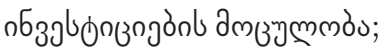

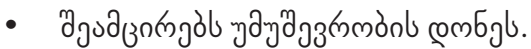

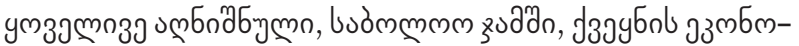

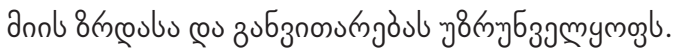

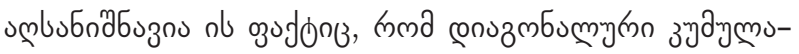

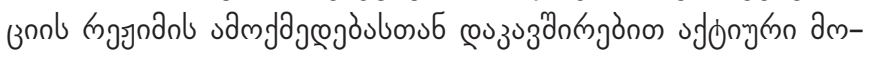

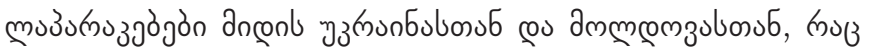

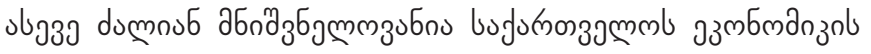
zodmngrigànbonzol.

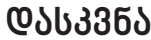

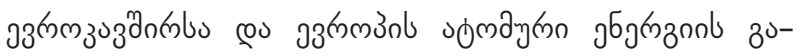

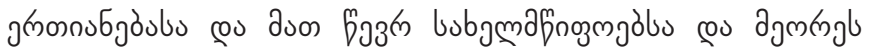

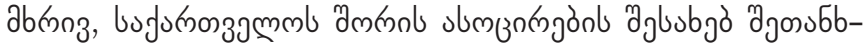

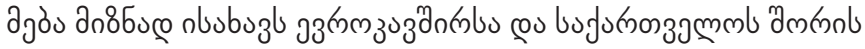

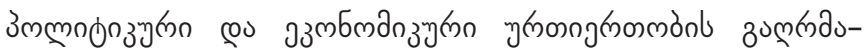

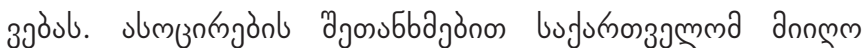

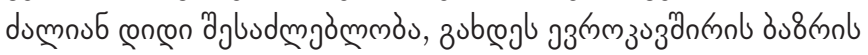

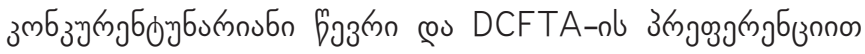

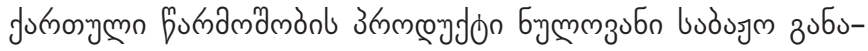

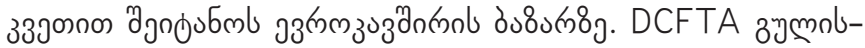

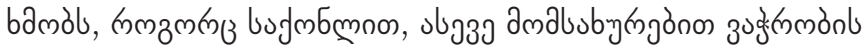

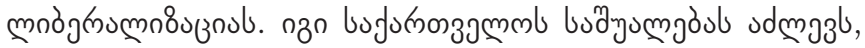

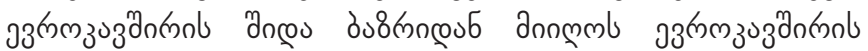

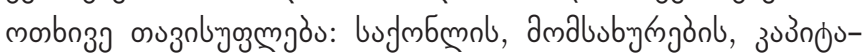

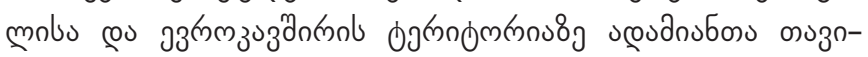

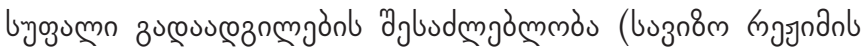

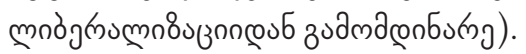

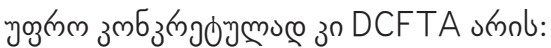

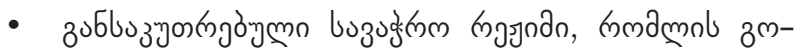

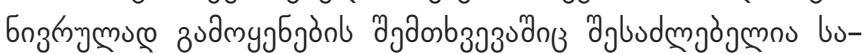

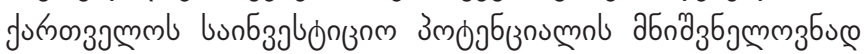

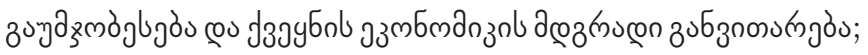

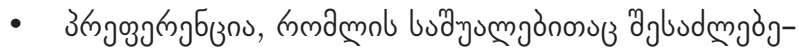

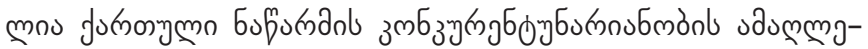

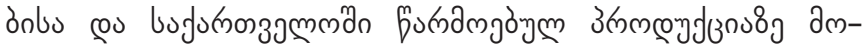

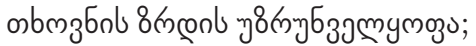

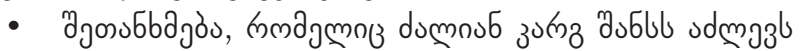

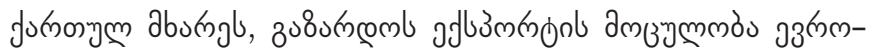

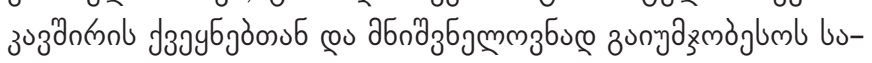

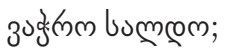

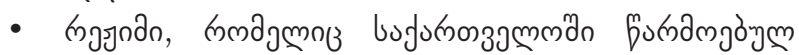

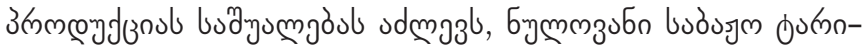

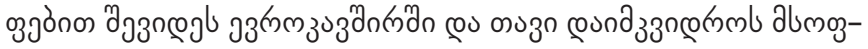

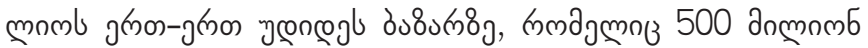

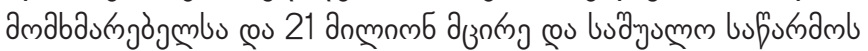
sgmonougall.

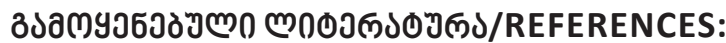

Association Agreement between Georgia, on the one hand, and the European Union and the European Atomic Energy Community, on the other, and their Member States. https://matsne.gov.ge/ka/document/view/2496959?publication=0 (In Georgian).

Data from the Deep and Comprehensive Free Trade Agreement (DCFTA) website data- http://www.dcfta.gov.ge/. (In Georgian). Data from the European Commission website- https://ec.europa.eu/info/index_en.

Data of the Ministry of Economy and Sustainable Development of Georgia - http://www.economy.ge/. (In Georgian).

Data of the National Statistics Office of Georgia - https://www.geostat.ge/ka. (In Georgian). 